\title{
Effect of exogenous adipose-derived stem cells in the early stages following free fat transplantation
}

\author{
YI YUAN, JIANHUA GAO and FENG LU \\ Department of Plastic and Reconstructive Surgery, Nanfang Hospital, Southern Medical University, \\ Guangzhou, Guangdong 510515, P.R. China
}

Received June 17, 2014; Accepted May 27, 2015

DOI: $10.3892 /$ etm.2015.2605

\begin{abstract}
Cotransplantation of adipose-derived stem cells (ASCs) is an effective therapeutic approach for enhancing the survival of transplanted fat tissue; however, the role of ASCs in free fat transplantation remains unclear. In the present study, fat harvested from C57BL/6 mice expressing green fluorescent protein was injected subcutaneously into the back of C57BL/6 mice, who also received ASCs (group A) or received the fat tissue only (group B). The grafts were harvested at days 1, 4, 7, 14, 30 and 90 following the transplantation. Graft volume and histology were evaluated, and the secretion levels of vascular endothelial growth factor (VEGF) and hepatocyte growth factor (HGF) were quantified using enzyme-linked immunosorbent assays. No statistically significant difference was identified between groups A and B in the graft survival rate up to day 14 following the aspirated fat transplantation; however, the graft survival rate decreased during the following 14-90 days. Initially, group =A exhibited a higher graft survival rate and a greater degree of angiogenesis compared with group B. The ratio of dead cells was not significantly different between the two groups on day 1; however, group A had a greater number of living interstitial cells compared with group B at the later time points. The secretion of VEGF by the ASCs had an earlier peak time in group A (day 4) compared with group B (day 7). In addition, the secretion of HGF in group A was greater compared with group B. Therefore, the role of exogenous ASCs in free fat transplantation may not directly participate in angiogenesis and adipogenesis, but may promote the survival ratio of the graft-resident interstitial cells, which are involved in angiogenesis and adipogenesis, via a paracrine effect.
\end{abstract}

Correspondence to: Professor Jianhua Gao or Professor Feng Lu, Department of Plastic and Reconstructive Surgery, Nanfang Hospital, Southern Medical University, 1838 Guangzhou Avenue North, Guangzhou, Guangdong 510515, P.R. China

E-mail: gaopsnf@pug.guangzhou.gd.cn

E-mail: doctorlufeng@hotmail.com

Key words: adipose-derived stem cells, fat transplantation, early stage, interstitial cells, paracrine effect, angiogenesis

\section{Introduction}

Fat transplantation is widely used in soft-tissue augmentation, such as hemifacial atrophy and facial rejuvenation, as well as nasolabial folds, and lip, chin, jaw and breast augmentation. Autologous fat tissue, a potentially ideal filler for soft-tissue defects, is safe and simple to harvest without producing scars on donor or recipient sites and without cross-infection or foreign-body reaction. However, the clinical outcomes of this technique are limited by a low graft survival rate (1-4). Previously, cytotherapy has been used in autologous fat transplantation. In one study, a novel strategy was developed, namely cell-assisted lipotransfer, in which the stromal vascular fraction containing adipose-drived stem cells (ASCs) was injected with the fat tissue. The results revealed improved graft retention compared with the control treatment (1). In addition, mixing ASCs with fat tissue prior to transplantation has been shown to enhance neovascularization and increase the survival rate of fat grafts (5). ASCs are known to secrete angiogenic growth factors, such as vascular endothelial growth factor (VEGF), hepatocyte growth factor (HGF) and basic fibroblast growth factor (bFGF), in response to injury, hypoxia and other conditions (6). VEGF and bFGF are considered to mobilize and recruit endothelial (progenitor) cells, subsequently accelerating the onset of angiogenesis (7). HGF is an additional important endothelial growth factor with potential angiogenic and mitogenic effects (8). Cotransplantation of ASCs with aspirated fat tissue may improve graft retention due to angiogenic effects. However, in vitro research has shown that ASCs are able to survive for several days under ischemic conditions (9). Therefore, the aim of the present study was to further elucidate the role of ASCs in the early stages following free fat transplantation.

\section{Materials and methods}

Cell isolation and culture. Ten 6 to 8-week-old green fluorescent protein (GFP)-expressing C57BL/6 mice weighing 18-23 g (Model Animal Research Center of Nanjing University, Nanjing, China) were selected, regardless of gender, for collection of ASCs. ASCs were isolated from the inguinal fat pad of C57BL/6 mice. The fat was washed with phosphate-buffered saline, excised and digested with $0.125 \%$ collagenase (Sigma-Aldrich, St. Louis, MO, USA) on a shaker at $37^{\circ} \mathrm{C}$ 
for $30 \mathrm{~min}$. An equal volume of Dulbecco's modified Eagle's medium (DMEM; Gibco Life Technologies, Carlsbad, CA, USA) with $10 \%$ fetal bovine serum (FBS; Gibco) was added to neutralize the collagenase. Subsequently, the cell suspension was filtered through a 200-mesh filter (Hebei Hongxia Medicine and Healthcare Product Co. Ltd., Hebei, China), and the mature adipocytes and connective tissue were separated from pellets by centrifugation at $1,200 \mathrm{x}$ g for $5 \mathrm{~min}$. The cell pellets were resuspended, plated at a density of $1 \times 10^{6}$ cells per $100-\mathrm{mm}$ dish in DMEM with $10 \% \mathrm{FBS}$, and cultured at $37^{\circ} \mathrm{C}$ in $5 \% \mathrm{CO}_{2}$. Primary cells were cultured for 7 days and were defined as passage 0 . The medium was replaced every 3 days, and the cells were passaged at a ratio of 1:3 per week. Only cells that had been cultured for three passages were used in the subsequent experiments.

Animal model and groups. Forty-six C57BL/6 mice (age, 6-8 weeks; weight, 18-23 g; Southern Medical University, Guangzhou, China) were selected regardless of gender for use as free fat transplantation models. The mice were anesthetized with $1 \%$ pentobarbital sodium $(45 \mathrm{mg} / \mathrm{kg})$. Ten of them were used to harvest inguinal fat pad, which was cut into pieces, similar to the size of aspirated fat tissue used for clinical fat injection in humans. The fat tissue samples were injected subcutaneously into the back of a C57BL/6 mouse using a $1 \mathrm{ml}$ syringe with a standardized blunt tipped 14 gauge infiltration cannula [the Coleman technique (10)]. Each C57BL/6 mouse was injected subcutaneously at two spots with fat tissue $(0.2 \mathrm{ml} / \mathrm{spot})$. In the experimental group, the fat tissue was mixed with $2 \times 10^{5}$ ASCs, while the control group received fat tissue only. At days 1, 4, 7, 14, 30 and 90 following fat transplantation, the grafts were excised and analyzed ( $n=6 /$ time-point). This study was approved by the Ethics Committee of Nanfang hospital (Southern Medical University, Guangzhou, China) and conducted in strict accordance with the recommendations in the Guide for the Care and Use of Laboratory Animals of the National Institutes of Health, and all efforts were made to minimize suffering.

Histology. Harvested adipose tissue samples were placed in $4 \%$ formalin and embedded in paraffin. Sections with a thickness of $4 \mathrm{~mm}$ were cut from the paraffin blocks. Specimens were stained with hematoxylin for $20 \mathrm{~min}$, rinsed with Scott's solution for $1 \mathrm{~min}$ and treated with $1 \%$ ammonia for $30 \mathrm{sec}$. Following incubation in $80 \%$ ethanol for $5 \mathrm{~min}$, the samples were stained with eosin for 2 min and dehydrated with a graded ethanol series. The samples were assessed under an Olympus BX51 microscope (Olympus, Tokyo, Japan) and photographed using an Olympus DP71 digital camera.

Whole-mount staining of fat grafts. Visualization of the fat grafts was performed using the procedure outlined by Eto et al (11). Accordingly, the grafts were cut into 0.5-1-mm pieces and incubated with Hoechst 33342 (Sigma-Aldrich) to stain all nuclei for $30 \mathrm{~min}$. Subsequently, the samples were incubated with propidium iodide (PI; S7112; Merck Millipore, Darmstadt, Germany) for 15 min to stain for dead cells. The samples were washed and observed directly with a confocal microscope system (FV1000 confocal microscope; Olympus).
Table I. Retention of the grafts in the two groups at different time points.

\begin{tabular}{cccc}
\hline Day & $\begin{array}{c}\text { Fat }+ \text { ASCs, ml } \\
(\mathrm{n}=6)\end{array}$ & $\begin{array}{c}\text { Fat only, ml } \\
(\mathrm{n}=6)\end{array}$ & P-value \\
\hline 1 & $0.13 \pm 0.01$ & $0.12 \pm 0.00$ & 0.272 \\
4 & $0.13 \pm 0.02$ & $0.13 \pm 0.01$ & 0.402 \\
7 & $0.15 \pm 0.01$ & $0.15 \pm 0.01$ & 0.905 \\
14 & $0.16 \pm 0.02$ & $0.16 \pm 0.02$ & 0.898 \\
30 & $0.13 \pm 0.02$ & $0.11 \pm 0.02$ & 0.006 \\
90 & $0.11 \pm 0.01$ & $0.09 \pm 0.01$ & 0.001 \\
\hline
\end{tabular}

Data are expressed as the mean \pm standard deviation. ASC, adipose-derived stem cells.

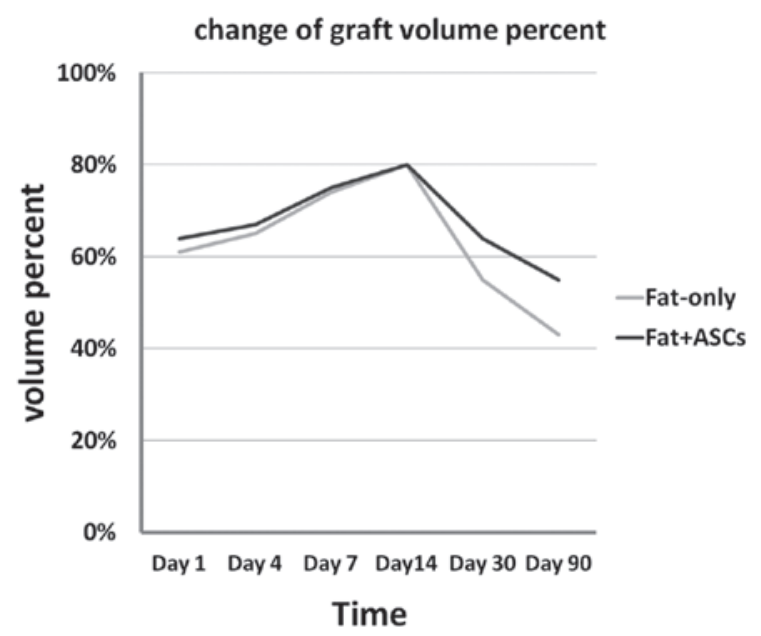

Figure 1. Nude mice received a fat transplant with ASCs or a fat transplant only, and the grafts were harvested at different times. The difference in the graft volume between the ASC and control groups was not statistically significant at every time-point; however, the graft volumes in the two groups were found to marginally decrease over time and after 14 days, the retention of grafts of the ASC group was higher than the control group. ASC, adipose-derived stem cells.

PI-positive cells were counted using four field images for each sample. Subsequently, the ratio of dead cells was calculated as follows: Number of PI- and GFP-positive cells/number of Hoechst- and GFP-positive cells.

Enzyme-linked immunosorbent assay (ELISA). Adipose tissue specimens were homogenized in lysis buffer (CWBiotech, Beijing, China), according to the manufacturer's instructions, and centrifuged at $12,000 \mathrm{x}$ g for $15 \mathrm{~min}$ at $4^{\circ} \mathrm{C}$. The aqueous layer was collected and assessed for VEGF and HGF secretion using Quantikine ELISA kits (R\&D Systems, Minneapolis, MN, USA), according to the manufacturer's instructions. After washing and aspirating five times with $400 \mu \mathrm{l}$ wash buffer, the plates were incubated for $2 \mathrm{~h}$ at room temperature with biotin-conjugated mouse VEGF (cat. no. MMV00) and mouse/rat HGF (cat. no. MHG00) primary antibodies, followed by a further five washes with $400 \mu \mathrm{l}$ wash buffer and incubation for $30 \mathrm{~min}$ at room temperature with horseradish peroxidase-conjugated 


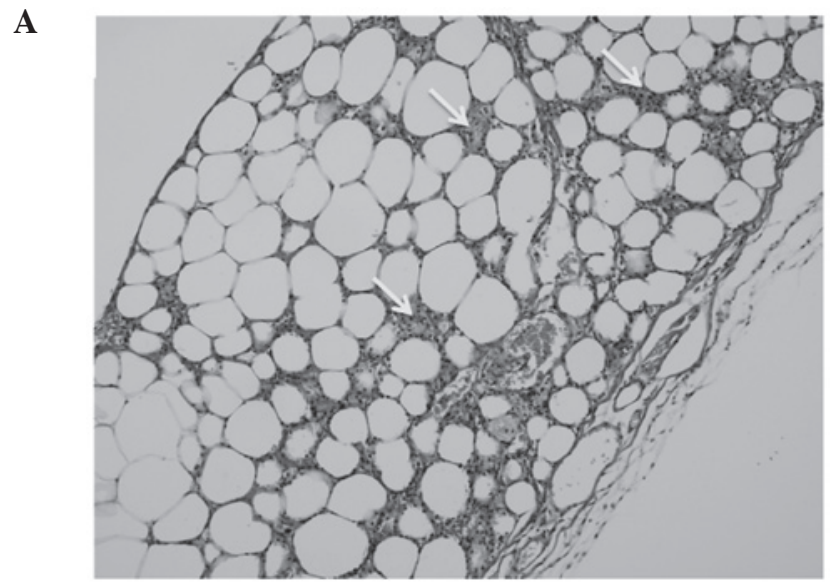

B

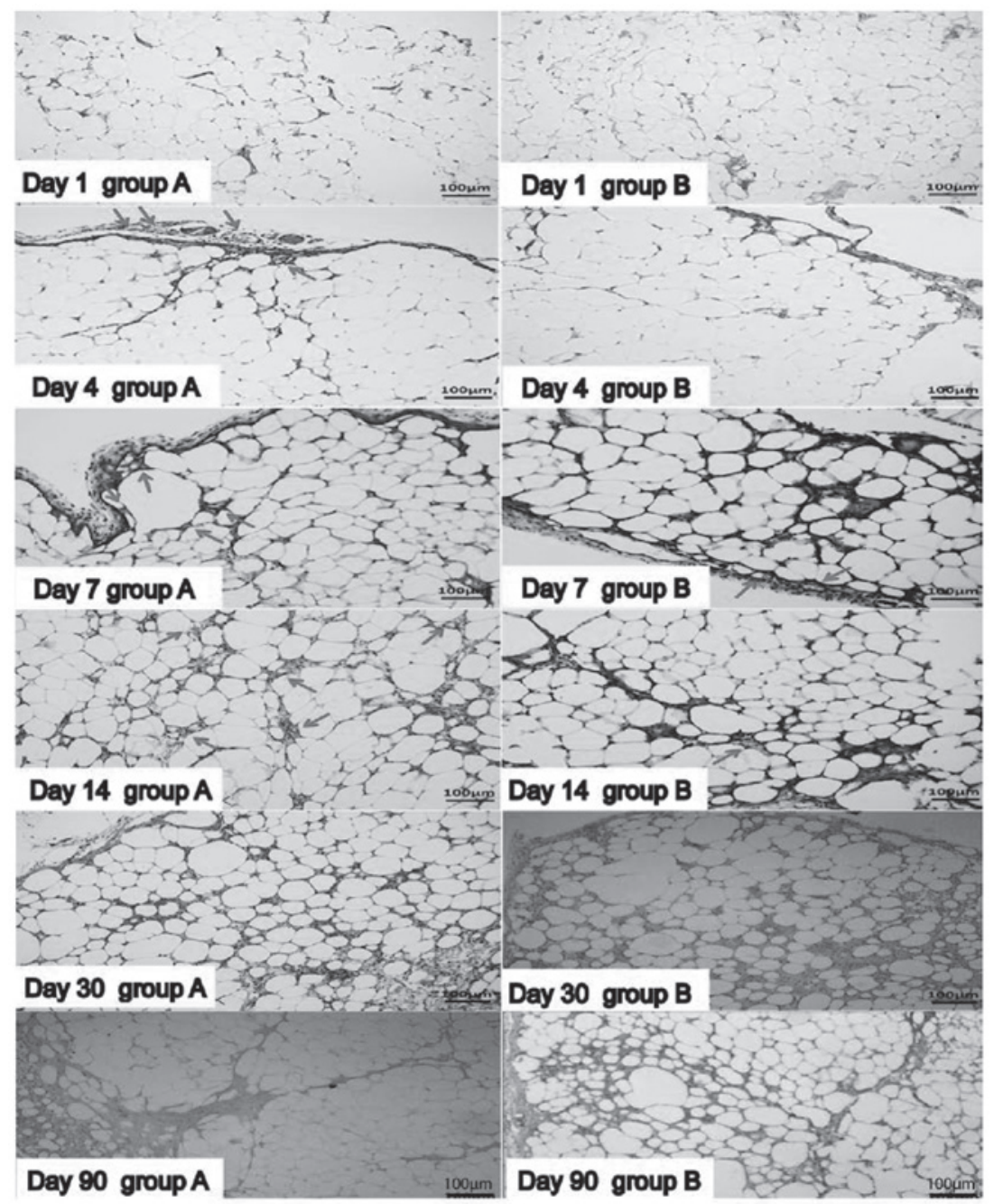

Figure 2. Fat grafts were sectioned, stained with hematoxylin and eosin, and imaged with a light microscope (magnification, x100). (A) Specimen from the fat-only group on day 14 . The arrows indicate the interstitial space between adipocytes and the infiltration of nucleated cells. (B) On day 4, blood vessels on the surface of the fat graft were observed in the fat + adipose-derived stem cell (ASC) group (group A); the vessels having grown into the graft from the surface of the graft. In the fat-only group (group B), this phenomenon appeared on day 7. The number of vessels in the grafts increased on day 14 in the two groups (red arrows indicate the vessels). The two groups presented with an increased number of infiltrated nucleated cells and a number of vacuoles on day 30 . In the fat + ASC group, the grafts were divided into a regular grid by fibrous tissue on day 90 ; however, the fat-only group exhibited an irregular structure and a greater extent of fibrous tissue.

streptavidin (Thermo Scientific Life Technologies, Carlsbad, CA, USA). The color was developed using the enzymatic substrate, $o$-phenylenediamine, and the optical density values of absorbance were measured on a microplate reader (Multiskan MK3; Thermo Scientific Life Technologies) at $450 \mathrm{~nm}$.
Statistical analysis. Data are expressed as the mean \pm standard deviation. SPSS software package version 17.0 (SPSS Inc., Chicago, IL, USA) was used for statistical analysis. A repeated measures analysis of variance was used to analyze the results. Furthermore, if the test revealed statistically significant differ- 

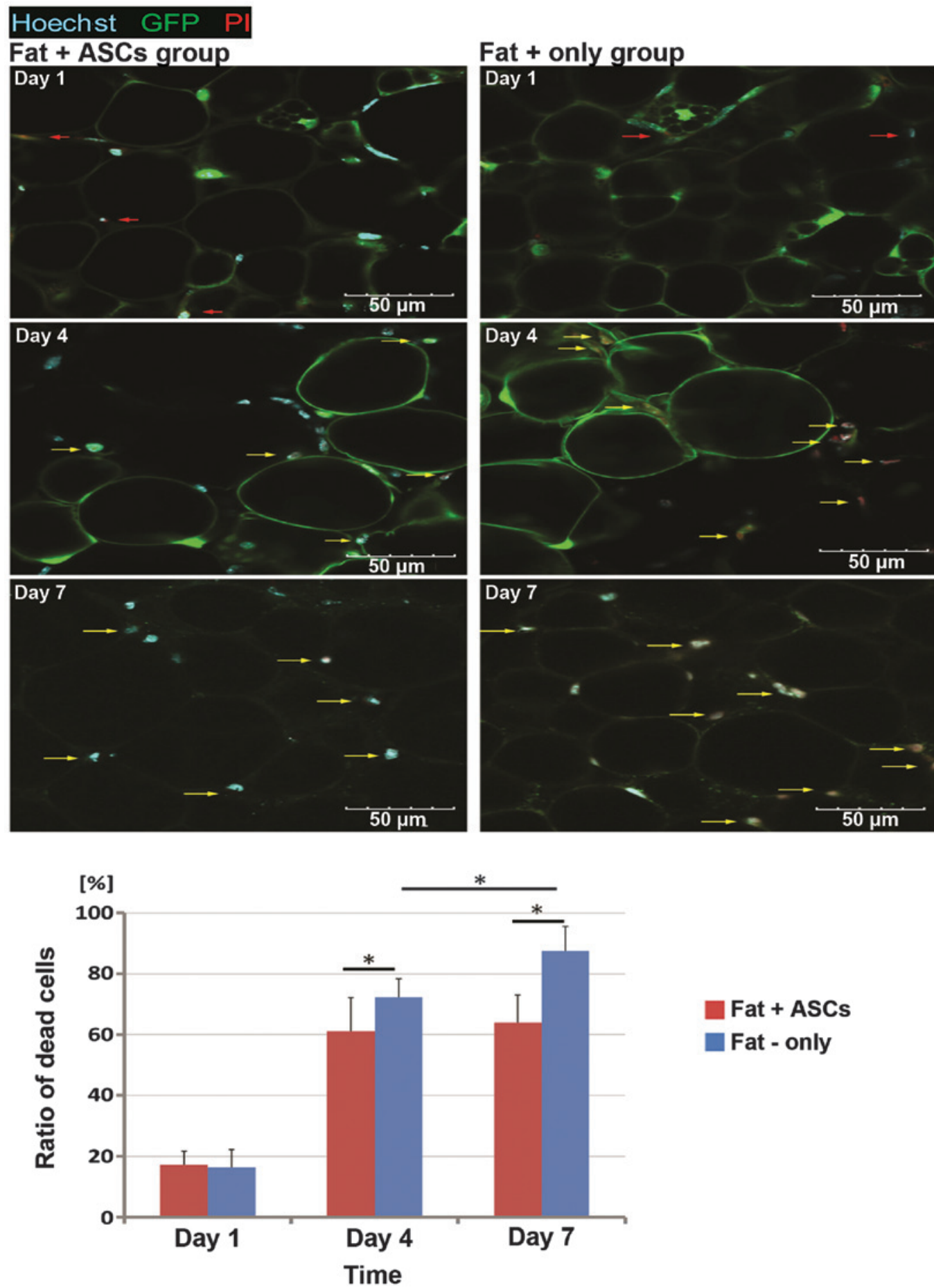

Figure 3. Whole-mount tissue staining with PI for the detection of dead cells. PI stained the nuclei of dead cells (red), Hoechst 33342 stained all the nuclei (blue) and GFP stained the cells of the graft (green). The dead cells (red arrows), the nuclei of which were localized within the ring-like GFP-positive area, may be the dead adipocytes. The dead cells (yellow arrows), the nuclei of which were localized in the gap of the ring-like GFP-positive areas, are the dead interstitial cells. " $\mathrm{P}<0.05(\mathrm{n}=6)$. PI, propidium iodide; GFP, green fluorescent protein; ASC, adipose-derived stem cell.

ences, a paired Student's t-test was used to compare the two groups at one time point, while one-way analysis of variance was used to compared one group over four time points. $\mathrm{P}<0.05$ was considered to indicate a statistically significant difference.

\section{Results}

Assessment of fat tissue survival. In the experimental group (group A), fat tissue was mixed with ASCs, while the control group (group B) received fat tissue only. Each mouse was injected with the two fat mixtures, with each of the two designated spots receiving one of the two fat mixtures in a random fashion. None of the animals succumbed during the study. At every time-point, the grafts of six mice were harvested by carefully removing them from the surrounding tissue. The volume of the grafts was measured using a liquid overflow method, which was subsequently used to calculate the survival ratio with the following formula: Survival volume/previous volume. 


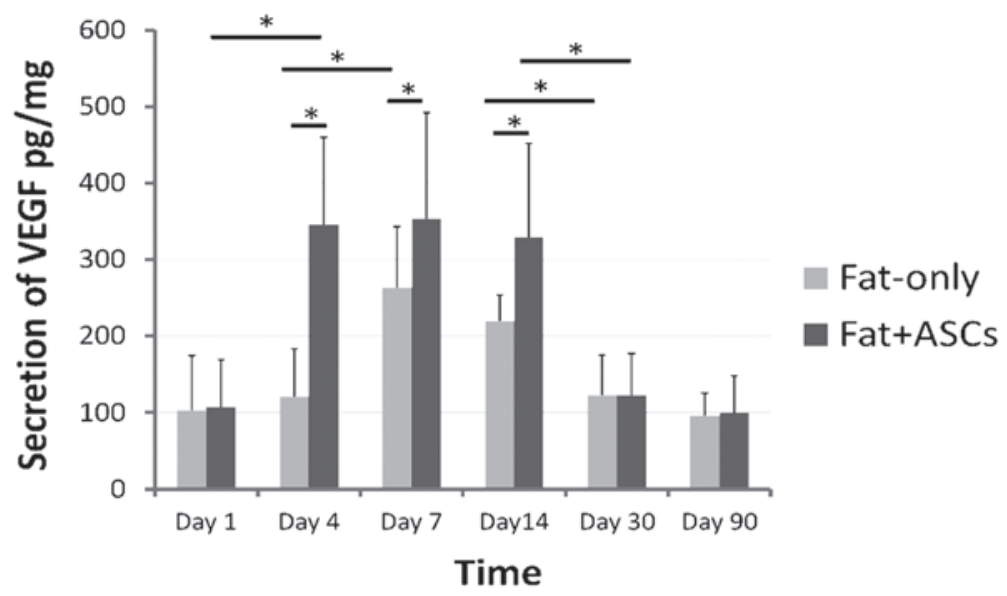

Figure 4. Enzyme-linked immunosorbent assay analysis for VEGF. The secretion of VEGF increased significantly on day 7 in the fat-only group, after which the secretion levels gradually decreased. By contrast, VEGF protein levels peaked between days 4 and 14 after the transplantation in the fat + ASCs group. The two groups exhibited low expression of VEGF after 30 days. ${ }^{*} \mathrm{P}<0.05(\mathrm{n}=6)$. VEGF, vascular endothelial growth factor; ASCs, adipose-derived stem cells.

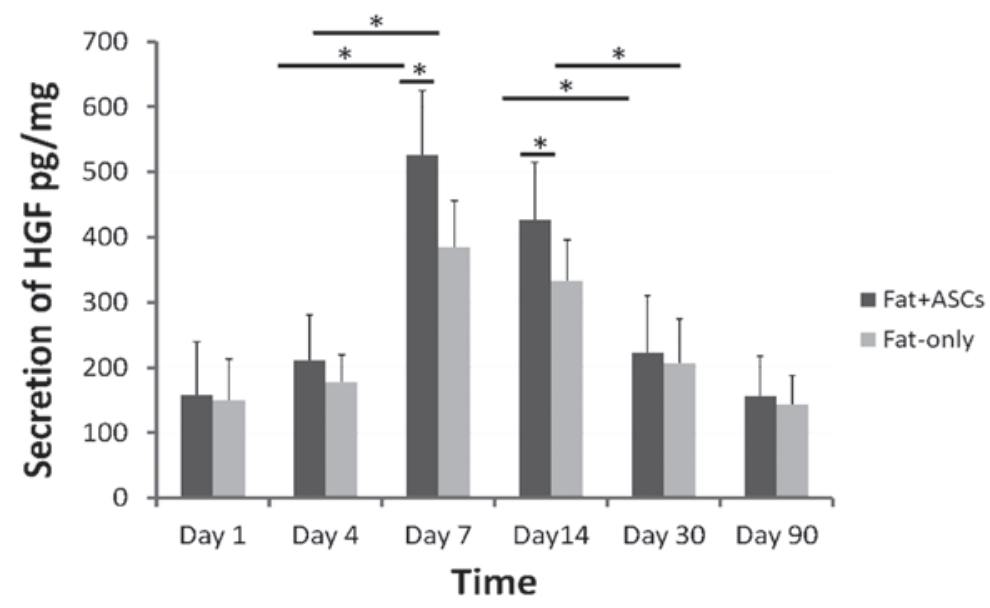

Figure 5. Enzyme-linked immunosorbent assay analysis for HGF. The secretion of HGF peaked on day 7 and decreased gradually between days 14 and 90 in the two groups. Group A exhibited increased levels of HGF secretion when compared with group B. "P<0.05 (n=6). HGF, hepatocyte growth factor; ASCs, adipose-derived stem cells.

The retention of grafts in the two groups is shown in Table $\mathrm{I}$. In addition, the survival ratios for groups $\mathrm{A}$ and $\mathrm{B}$ are shown in Fig. 1. The difference in the graft volumes between the ASC and control groups was not statistically significant until day 14 following the aspirated fat transplantation, although the graft volumes in the two groups were shown to marginally increase over time up to day 14 and, after 14 days, the retention of grafts of the ASC group was higher than the control group. However, the graft survival rate notably decreased between days 14 and 30 , and continued to decrease gradually during the 30-90 day period. The fat + ASCs group exhibited a higher graft survival rate compared with the fat-only group.

Histological evaluation of grafts. Histological evaluation revealed that a number of the adipocytes decreased in size and increased in number over time. On day 14, gaps between the adipocytes were observed, and infiltrated nucleated cells became immersed in the interstitial space between adipocytes in the two groups (Fig. 2A). On day 4, blood vessels on the surface of the fat graft were observed in the fat + ASCs group. In addition, vessels were shown to grow into the graft from the surface of the graft. However, in the fat-only group, this phenomenon appeared on day 7. The number of vessels in the grafts increased on day 14 in the two groups (Fig. 2B); however, the fat + ASCs group exhibited an increased number of blood vessels compared with the control group. There was no evident fibrosis in either group in the early stages following the aspirated fat transplantation. The two groups presented an increasing number of infiltrated nucleated cells and a number of vacuoles on day 30 . The size of the adipocytes varied. In the fat + ASCs group, the grafts were divided into a regular grid by fibrous tissue on day 90 ; however, the fat-only group exhibited an irregular structure with increased fibrous tissue.

Whole-mount staining of the fat grafts. The ratio of PI-positive dead cells to the total cells (without transplant ASCs) increased from day $1(16.40 \pm 5.79 \%)$ following the transplantation, with a sharp increase at day $4(72.4 \pm 11.11 \%)$ and further increments at later time points (day 7, 87.48 $\pm 8.11 \%$ ) in group B (Fig. 3B). The ratio of dead cells showed no statistically significant difference between the two groups on day 1 (group A, 17.22 $\pm 4.44 \%$ ). However, fewer dead cells were observed in 
group A compared with group B at the later time points (day 4, $61.07 \pm 5.94 \%$; day 7,64.05 $\pm 9.03 \%$ ). No statistically significant difference in the dead cell ratio was observed between days 4 and 7 in group A (Fig. 3A). The green fluorescence became weaker over time in the two groups.

Expression of VEGF and HGF. In group B, the ELISA results demonstrated that the secretion of VEGF increased significantly on day 7, after which the levels decreased gradually. By contrast, VEGF protein levels peaked between days 4 and 14 following transplantation in group A (Fig. 4), which indicated that the secretion of VEGF by the ASCs had an earlier peak time and higher level, as shown by the comparison between group A and group B. The two groups exhibited low expression levels of VEGF after 30 days.

Following transplantation, the secretion of HGF peaked on day 7 and decreased gradually between days 14 and 90 in the two groups (Fig. 5). However, higher levels of HGF secretion were observed in group A when compared with group B.

\section{Discussion}

A number of studies have focused on ASCs as helper cells in autologous fat transplantation. Lu et al (5) reported that ASCs significantly increase the transplant survival rate $(60.1 \pm 17.6 \%)$,

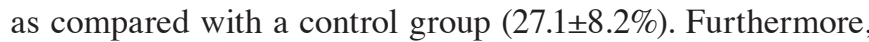
in a clinical study, Yoshimura et al (1) used ASC-assisted lipotransfer for facial lipoatrophy and obtained improved clinical results.

However, in contrast to these studies, the present study revealed that there was no statistically significant difference between the fat-only and the fat + ASCs groups in the graft survival rate immediately following the aspirated fat transplantation (days 1-14). The results indicated that the difference between the two groups was observed in the graft tissue structure rather than the graft survival rate. With regard to the histology examination, group A exhibited increased vascularization compared with group B at days 4, 7 and 14 .

The graft survival rate of the two groups decreased markedly during days 14-30, and continued to decrease gradually between days 30 and 90 . However, the fat + ASCs group had a higher survival rate compared with the fat-only group, which resulted from the difference in the histological structure between the two groups prior to day 14. Necrosis of the fat tissue is primarily observed on days 3 and 7 under ischemic conditions (12). Therefore, the decrease in the graft volume between days 14 and 90 may be due to the absorption of necrotic tissue and fibrosis following necrosis. Adipogenesis and angiogenesis are exerted by activated adipose stem/progenitor cells, and completion of these processes requires several months. A number of the dead adipocytes are replaced with new next-generation adipocytes during the first three months while others are not (13). Lipid droplets are absorbed by macrophage phagocytosis, although the absorption is very slow and the absorption period is dependent on the diameter of the lipid droplets; when the lipid droplet diameter was large (e.g. $10 \mathrm{~mm}$ ), the cyst wall was formed before completing absorption and the cyst wall calcified over time.

A previous study offered insight into the histological changes of graft fat (14). On day 1 following the fat injection, the number of viable adipocytes is markedly decreased, and the viable zone is restricted to the peripheral area $(<300 \mu \mathrm{m}$ from the edge) (9). In the present study, the whole-mount stained images show a degree of cell death on day 1 , with the nucleus of the dead cells localized within the ring-like GFP-positive area. A previous study indicated that excised adipose tissue contained 10-15\% adipocytes (11). The majority of the dead cells on day 1 may represent the dead adipocytes. The green fluorescence became weaker over time, which may be due to an increase in adipocyte necrosis, leading to GFP degradation.

On day 4, graft angiogenesis occurs from the host bed vascular system (15). A similar result was obtained in the present study, and the histological evaluation of the fat graft indicated that the mice that received ASCs had a more highly vascularized fat graft compared with the control mice at days 4, 7 and 14. In addition, an increased number of inflammatory cells were observed in the area of high-density neovascularization. This phenomenon may be partly associated with macrophages, which promote angiogenesis. Additional studies have indicated that macrophages appear to undergo marked phenotypic changes in tumors when exposed to hypoxia, and activate the expression of a number of mitogenic and proangiogenic cytokines and enzymes (16). Regenerative adipogenic changes, such as an increasing number and size of new adipocytes, initiate from the peripheral dead adipocyte area at day 7 following fat transplantation (9). In the present study, smaller adipocytes, as compared with normal, were observed after day 7. The small adipocytes were hypothesized to be derived from the surviving interstitial cells (including the graft-resident ASCs that contribute to adipogenesis), host-derived precursors or the dedifferentiated adipocytes; however, further research is required to indicate the origin of the small adipocytes. In the later stages following fat transplantation, fibrous tissue growth occurred instead of adipocyte necrosis. Nevertheless, there was no evident fibrosis in either group during the early stages following the fat transplantation.

The results of the present study demonstrated that ASCs enhanced the density of neovascularization, although this had no effect on the survival rate of the graft fat in the early stages following transplantation. Angiogenic growth factors secreted by ASCs, such as VEGF and HGF, may be responsible for this finding. The injection force mechanically injures the fat, and non-vascularization causes ischemic injury. ASCs can secrete various angiogenic growth factors, such as VEGF, HGF and bFGF, in response to injury, hypoxia and other conditions (6). Lee et al (17) indicated that ASC proliferation and secretion of angiogenic growth factors, such as VEGF and bFGF, were significantly increased under hypoxic conditions $\left(2 \% \mathrm{O}_{2}\right)$. Similarly, Rubina et al (18) reported that the mRNA expression levels of angiogenic growth factors, such as VEGF, HGF and bFGF, increased from 1.7 to 4.1 -fold in response to hypoxia. Furthermore, an additional study that used a Wister rat model of free fat grafts indicated that VEGF was expressed in the interstitial mononuclear cells, predominantly on day 7 , after which the levels decreased (19), which was confirmed in the present experiments. However, in the current study, the ASCs caused VEGF secretion to peak earlier in the fat + ASCs group on day 4. HGF is an additional important endothelial growth factor with potential angiogenic and mitogenic effects (8). 
ASCs maintained the secretion of HGF at a high level following transplantation. Following mechanical injury and ischemia-reperfusion injury to human adipose tissue, fibroblast growth factor-2, epidermal growth factor, transforming growth factor- $\beta$ and platelet-derived growth factor are secreted during the early stages of wound healing (days $0-1$ ). Thereafter, as the levels of these growth factors decrease, VEGF and HGF secretion gradually increases between days 5 and 7 post-injury (19). In the present study, the transplantation of ASCs resulted in increased VEGF and HGF secretion, which caused VEGF levels to peak earlier and maintain the secretion of HGF at a higher level. ASCs may contribute to angiogenesis by secreting angiogenic growth factors, which appear in the early stages following aspirated fat transplantation. In vitro studies have revealed that ASCs remain viable for only three days under ischemic conditions (9). Since free fat transplantation causes an ischemic condition, cell death occurs in the majority of the transplanted ASCs during the first week following transplantation. Subsequently, angiogenesis is initiated at $\sim$ day 4 when the majority of adipocytes have undergone cell death (15). Due to the greater hypoxia tolerance of ASCs compared with adipocytes and the earlier initiation of angiogenesis caused by the transplanted ASCs, there were $\sim 43 \%$ of interstitial cells surviving at day 4 in group A. However, $<30 \%$ of interstitial cells had survived at day 4 in group B, and the number of living interstitial cells continued to decrease to day 7 . The transplanted ASCs prevented the further increase in interstitial cell death and promoted angiogenesis from the host sooner by secreting angiogenic growth factors, which contributed to the survival of interstitial cells (including the graft-resident ASCs).

In conclusion, exogenous ASCs may not directly participate in angiogenesis and adipogenesis following free fat transplantation, but instead may promote the survival ratio of the graft-resident interstitial cells via a paracrine effect, which are involved in angiogenesis and adipogenesis. The preliminary results suggest that exogenous ASCs are effective in fat transplantation, which provides an experimental basis for further research and clinical work.

\section{Acknowledgements}

The study was supported by grants from the National Natural Science Foundation of China (grant nos. 81171834 and 81372083) and the Science and Technology Key Program of Guangzhou, China (grant no. 11C32120716).

\section{References}

1. Yoshimura K, Sato K, Aoi N, et al: Cell-assisted lipotransfer for facial lipoatrophy: Efficacy of clinical use of adipose-derived stem cells. Dermatol Surg 34: 1178-1185, 2008.

2. Yoshimura K, Asano Y, Aoi N, et al: Progenitor-enriched adipose tissue transplantation as rescue for breast implant complications. Breast J 16: 169-175, 2010.

3. Longobardi G, Pellini E, Diana G and Finocchi V: Rhytidectomy associated with autologous fat transplantation in Parry-Romberg syndrome. J Craniofac Surg 22: 1031-1034, 2011.

4. Sykes JM, Tapias V and Pu LL: Autologous fat grafting viability: Lower third of the face. Facial Plast Surg 26: 376-384, 2010.

5. Lu F, Li J, Gao J, et al: Improvement of the survival of human autologous fat transplantation by using VEGF-transfected adipose-derived stem cells. Plast Reconstr Surg 124: 1437, 2009.

6. Yoshimura K, Suga H and Eto H: Adipose-derived stem/progenitor cells: Roles in adipose tissue remodeling and potential use for soft tissue augmentation. Regen Med 4: 265-273, 2009.

7. Hirschi KK, Skalak TC, Peirce SM and Little CD: Vascular assembly in natural and engineered tissues. Ann N Y Acad Sci 961: 223-242, 2002.

8. Morishita R, Sakaki M, Yamamoto K, et al: Impairment of collateral formation in lipoprotein(a) transgenic mice: Therapeutic angiogenesis induced by human hepatocyte growth factor gene. Circulation 105: 1491-1496, 2002.

9. Eto H, Kato H, Suga H, et al: The fate of adipocytes after non-vascularized fat grafting: Evidence of early death and replacement of adipocytes. Plast Reconstr Surg 129: 1093-1095, 2012.

10. Coleman SR: Facial recontouring with lipostructure. Clin Plast Surg 24: 347-367, 1997.

11. Eto H, Suga H, Matsumoto D, et al: Characterization of structure and cellular components of aspirated and excised adipose tissue. Plast Reconstr Surg 124: 1087-1097, 2009.

12. Suga H, Eto H, Aoi N, et al: Adipose tissue remodeling under ischemia: Death of adipocytes and activation of stem/progenitor cells. Plast Reconstr Surg 126: 1911-1923, 2010.

13. Yoshimura K, Eto H, Kato H, et al: In vivo manipulation of stem cells for adipose tissue repair/reconstruction. Regen Med 6: 33-41, 2011.

14. Rieck B and Schlaak S: Measurement in vivo of the survival rate in autologous adipocyte transplantation. Plast Reconstr Surg 111: 2315-2323, 2003.

15. Billings E Jr and May JW Jr: Historical review and present status fo free fat graft autotransplantation in plastic and reconstructive surgery. Plast Reconstr Surg 83: 368-381, 1989.

16. Murdoch C, Giannoudis A and Lewis CE: Mechanisms regulating the recruitment of macrophages into hypoxic areas of tumors and other ischemic tissues. Blood 104: 2224-2234, 2004.

17. Lee EY, Xia Y, Kim WS, et al: Hypoxia-enhanced wound-healing function of adipose-derived stem cells: Increase in stem cell proliferation and up-regulation of VEGF and bFGF. Wound Repair Regen 17: 540-547, 2009

18. Rubina K, Kalinina N, Efimenko A, et al: Adipose stromal cells stimulate angiogenesis via promoting progenitor cell differentiation, secretion of angiogenic factors, and enhancing vessel maturation. Tissue Eng Part A 15: 2039-2050, 2009.

19. Nishimura T, Hashimoto H, Nakanishi I and Furukawa M: Microvascular angiogenesis and apoptosis in the survival of free fat grafts. Laryngoscope 110: 1333-1338, 2000. 